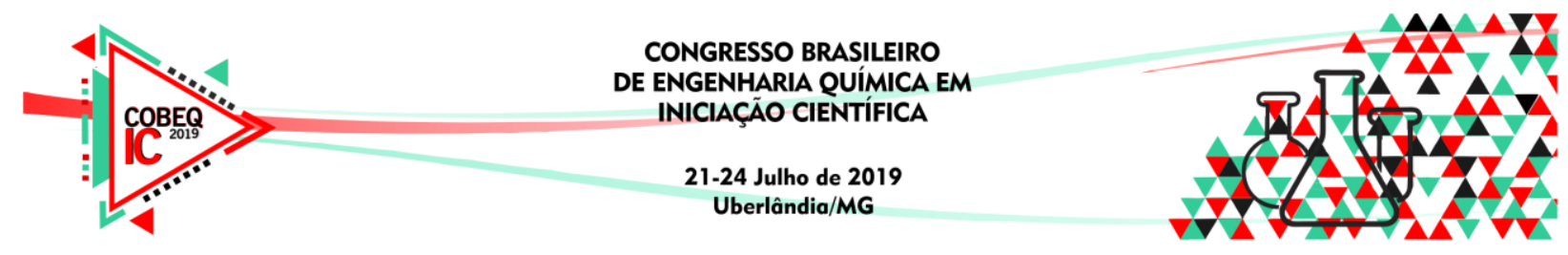

\title{
USO DE FERTILIZANTE ORGANOMINERAL DE LIBERA- ÇÃO GRADUAL DE NUTRIENTES NA CULTURA DE SOJA
}

\author{
R. T. X. SOUZA1, B. C. M. HENRIQUE ${ }^{2}$, L. C. M. HENRIQUE ${ }^{3}$ e H. M. HENRIQUE ${ }^{4}$ \\ ${ }^{1}$ Universidade Federal de Uberlândia, Instituto de Ciências Agrárias \\ ${ }^{2}$ Universidade Federal de Uberlândia, Faculdade de Engenharia Elétrica \\ ${ }^{3}$ Universidade Federal de Uberlândia, Faculdade de Engenharia Química \\ ${ }^{4}$ Universidade Federal de Uberlândia, Faculdade de Engenharia Mecânica \\ E-mail para contato: humberto@ufu.br
}

\begin{abstract}
RESUMO - O objetivo deste trabalho foi investigar o efeito do uso de fertilizante organomineral produzido a partir de resíduo de torta de filtro de usinas sucroenergéticas sobre a produtividade de campo de soja em relação à adubação mineral convencional. $\mathrm{O}$ experimento foi conduzido no município de Tupacigura/MG seguindo um delineamento de blocos casualizados com quatro repetições. As variáveis analisadas foram a produtividade, teor foliar de NPK. Houve efeito estatístico do fertilizante organomineral sobre a produtividade da soja e sobre a absorção de $\mathrm{N}$ e $\mathrm{P}$ por essa cultura. A produtividade da soja nos tratamentos que receberam o organomineral foi superior à do tratamento mineral.
\end{abstract}

\section{INTRODUÇÃO}

A concentração de nutrientes nos adubos orgânicos é baixa para atender as necessidades das culturas e, por isso, é necessário a aplicação de grande quantidade desses adubos. Já o uso de adubos tipo organomineral é mais viável, pois pode ser aplicado em menores quantidades por área. Outra vantagem da utilização de organomineral em relação ao fertilizante mineral é o fornecimento de matéria orgânica para o solo, o que propicia melhor desenvolvimento das plantas. Bhattacharyya et al. (2008) relataram que as culturas da soja e do trigo cultivadas em sucessão responderam à aplicação de NPK fornecido via fertilizantes minerais, mas o rendimento máximo foi obtido com o NPK com esterco bovino. Ghosh et al. (2009) trabalhando com consórcio soja/sorgo sob seis combinações de adubação orgânica e mineral, observaram que a aplicação de $75 \%$ NPK mineral recomendado mais esterco de galinha ou esterco bovino foi a melhor combinação. O objetivo deste experimento foi comparar a eficiência agronômica de um organomineral aplicado em doses reduzidas em relação ao fertilizante mineral.

\section{O FERTILIZANTE ORGANOMINERAL DESENVOLVIDO}

O fertilizante organomineral utilizado neste trabalho usa matéria orgânica proveniente de tratamento de resíduos da agroindústria sucroenergética (torta de filtro) por processo de compostagem assistida e sua posterior transformação em organomineral. O resíduo passa por uma caracterização química, física e microbiológica. Os resultados são usados na elaboração de um protocolo de ações que envolve a aplicação de nutrientes, meio de cultura e inóculos 


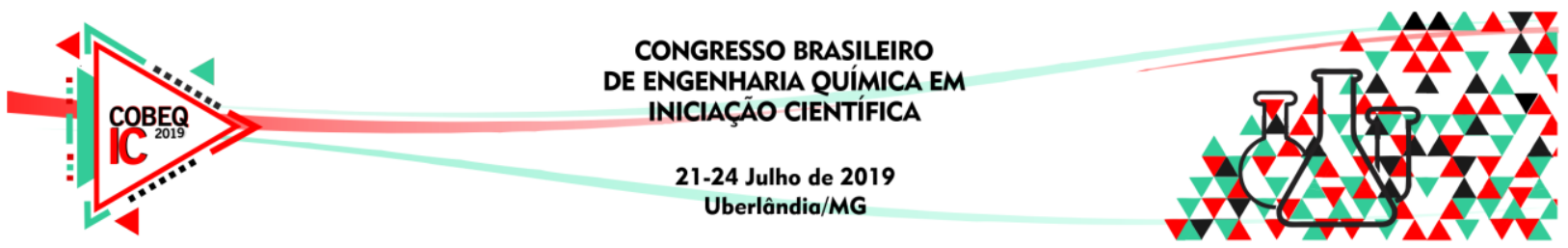

microbianos que são adicionados ao resíduo para sua transformação. O processo termofílico se encerra entre 20 e 40 dias solubilizando nutrientes e descontaminando o resíduo. $\mathrm{O}$ material obtido é usado na fabricação do organomineral que inclui a adição de um polímero orgânico biodegradável que associado às condições adequadas de temperatura e pressão faz com que os nutrientes minerais adicionados se agreguem à matriz orgânica porosa formando um pellet que adquire a capacidade de liberar os nutrientes progressivamente de acordo com a capacidade de absorção das plantas. O principal diferencial do fertilizante é a liberação gradual de nutrientes provocado pelo encapsulamento dos minerais na matriz orgânica. À medida que a microbiota do solo é ativada pela matéria orgânica do organomineral os nutrientes vão sendo liberados para as plantas. Esse processo garante expressiva redução da perda de nutrientes dos fertilizantes tradicionais minimizando as perdas por volatilização de nitrogênio, fixação de fósforo e lixiviação de potássio e nitratos reduzindo a contaminação de lençóis freáticos e a emissão de gases do efeito estufa, bem como o uso racional dos insumos minerais diminuindo o seu impacto ambiental. Com essa tecnologia, a produção de fertilizante organomineral ganhou escala, reduziu custos e se tornou competitiva em relação ao processo tradicional. A Figura 1 mostra uma foto aérea de unidade fabril e a Figura 2 mostra detalhes do tratamento de resíduos pelo processo de compostagem assistida desenvolvido.

A tecnologia foi reconhecida como sustentável, foi selecionada e recebeu apoio do Massachussetts Institute of Technology (MIT) através do Global Entrepreneurship Laboratory (G-Lab). Posteriormente, a Finep apoiou o desenvolvimento da tecnologia em projetos de Subvenção Econômica e Inova Brasil, que resultou na solução inovadora, ambientalmente correta, agronomicamente viável, economicamente rentável e socialmente responsável validada em campo. Essa tecnologia foi premiada com o $2^{\circ}$ lugar do Prêmio FINEP de Inovação na categoria Inovação Sustentável na Região Sudeste com o projeto "Tratamento de Resíduos Sólidos e Produção de Fertilizante - Visando a inserção sustentável de resíduos no ciclo produtivo e seu uso na agricultura".

Além do processo de tratamento de resíduos orgânicos, o processo desenvolvido continua com a fabricação do organomineral que inclui a aplicação de um polímero orgânico biodegradável que associado às condições adequadas de temperatura e pressão que faz com que os nutrientes minerais adicionados se agreguem à matriz orgânica porosa formando um pellet que adquire a capacidade de liberar os nutrientes progressivamente de acordo com a capacidade de absorção das plantas. Essas inovações atuam nos principais mecanismos de deficiências da adubação tradicional. Os principais tributos inovativos são:

1. Possui NPK, matéria orgânica bioestabilizada e micronutrientes no mesmo pellet;

2. Apresenta propriedades mecânicas superiores(alta dureza, baixa geração de pó e uniformidade);

3. Liberação gradual de nutrientes;

4. Redução das perdas dos nutrientes por volatilização, fixação, lixiviação;

5. Distribuição homogênea de nutrientes no solo

6. Aumenta a eficiência de absorção de NPK pela planta

7. Protege o solo da salinização por altos níveis de adubação mineral

8. Viabiliza a aplicação da matéria orgânica e micronutrientes em $100 \%$ da área plantada. 


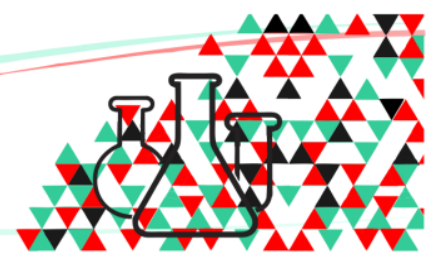

Figura 1 - Foto aérea de unidade fabril mostrando os galpões de compostagem assistida ao fundo e galpões de produção do organomineral em primeiro plano.

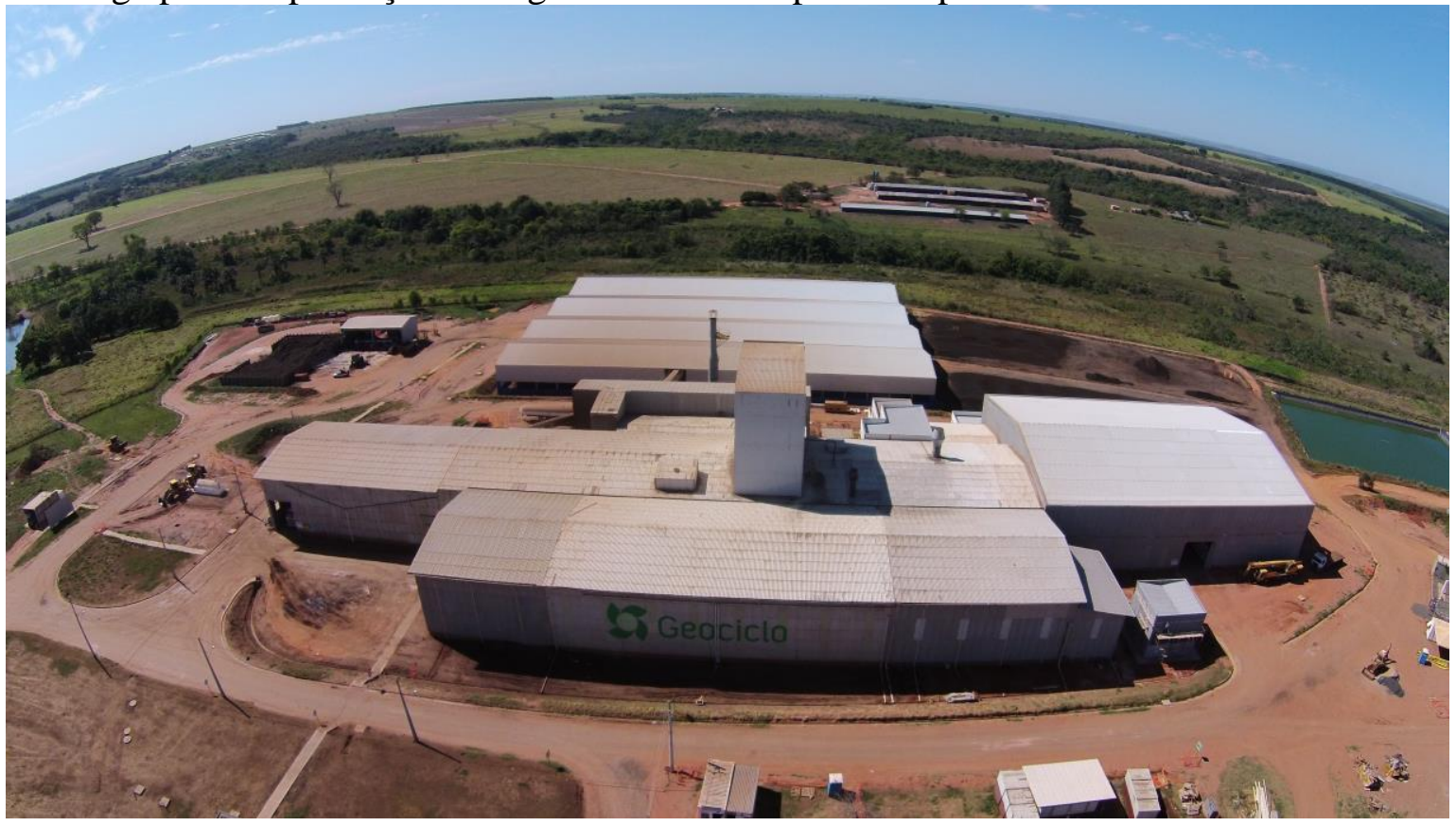

Figura 2 - Detalhes do tratamento de resíduos pelo processo de compostagem assistida.

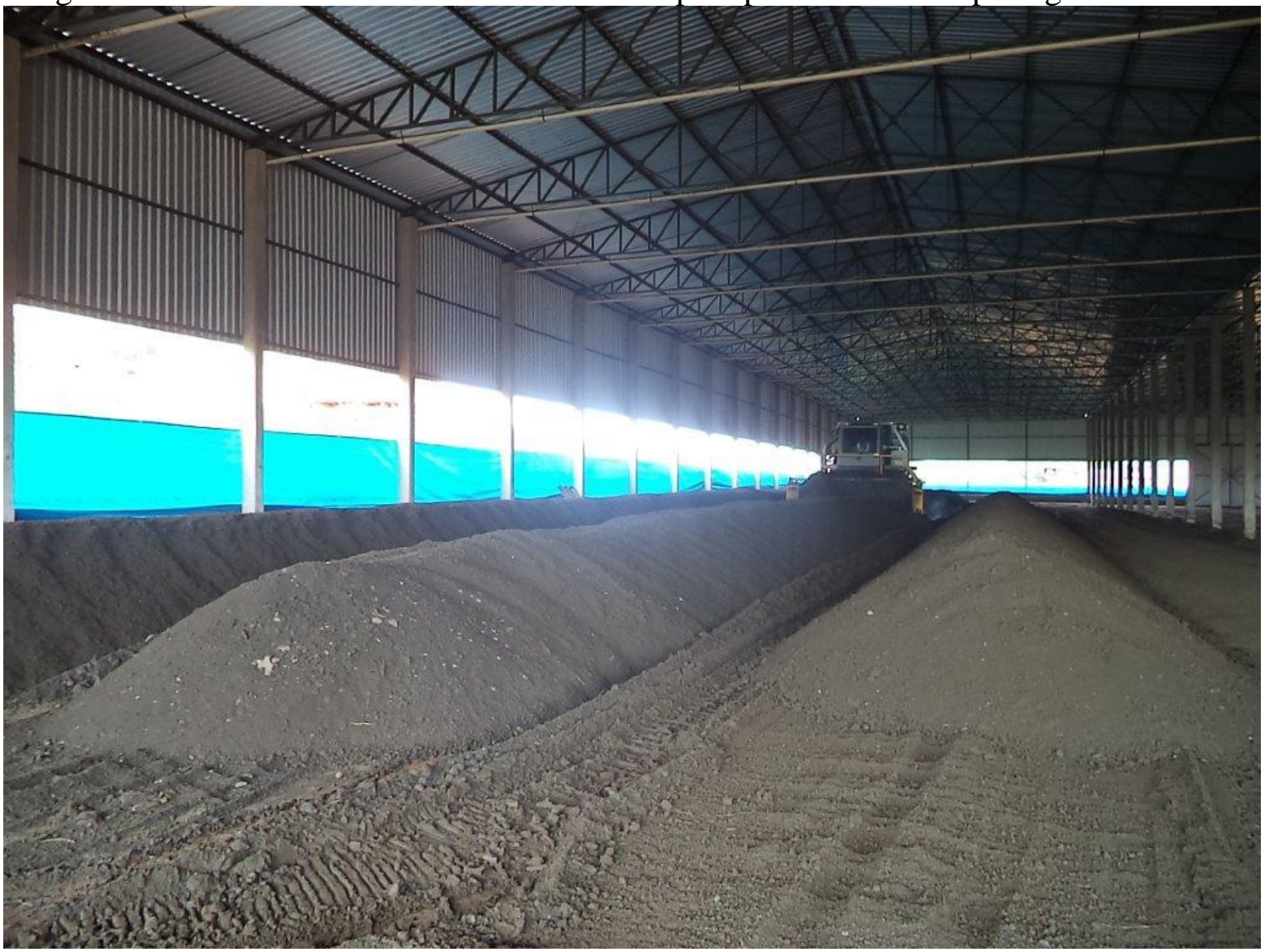




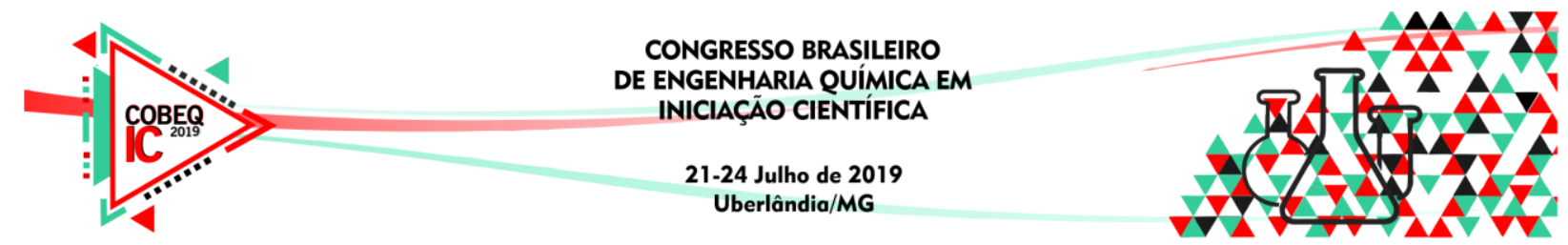

\section{MATERIAIS E MÉTODOS}

Devido à falta de espaço nesse artigo para mais detalhes dos aspectos do processo de produção do fertilizante proposto, optou-se aqui por apresentar alguns resultados experimentais que suportam as evidências de maior eficiência agronômica do fertilizante organomineral em relação ao fertilizante convencional (mineral).

O experimento foi instalado na fazenda Estância HZ no município de Tupaciguara/MG e a cultivar de soja utilizada foi a "Valiosa". O delineamento experimental utilizado foi o de blocos casualizados com quatro repetições. Os tratamentos foram: o controle (sem fertilizante na semeadura), fertilizante mineral (04-20-20) na dose de $400 \mathrm{~kg} / \mathrm{há}$, fertilizante organomineral (03-15-15) nas doses de 214, 320, 427 e $543 \mathrm{~kg} / \mathrm{ha}$. Essas doses de organomineral representam respectivamente $40,60,80$ e $100 \%$ de NPK em relação ao tratamento mineral. Cada parcela experimental foi composta de oito linhas de cultivo espaçadas de $0,5 \mathrm{~m}$ com 100 metros de comprimento, totalizando $400 \mathrm{~m}^{2}$. O solo da área experimental foi classificado como Latossolo Vermelho de textura média e com $\mathrm{pH}$ em água de 5,$3 ; 49 \mathrm{mg} / \mathrm{dm}^{3}$ de P (resina), $155 \mathrm{mg} / \mathrm{dm}^{3}$ de K; 1,9 cmol $/ \mathrm{dm}^{3}$ de Ca, 0,9 cmolc. $\mathrm{dm}^{3}$ de $\mathrm{Mg}, 2,2 \mathrm{cmol} / \mathrm{dm}^{3}(\mathrm{H}+\mathrm{Al}), 59 \%$ de saturação por bases $(\mathrm{V}), 3,3 \mathrm{dag} / \mathrm{kg}$ de matéria orgânica. Não foi necessário fazer calagem antes do plantio pois a saturação de bases estava maior que 50\%. A aplicação dos tratamentos foi feita no momento da semeadura direta da soja com uma semeadora com tração mecanizada. O stand utilizado foi de 18 plântulas por metro. A colheita foi realizada 130 dias após a semeadura. As variáveis analisadas foram a produtividade e os teores foliares de nitrogênio, fósforo e potássio que foram submetidas à análise de variância com auxílio do software Sisvar® (FERREIRA, 2008) e do software SPSS Inc. released 2008.

\section{RESULTADOS E DISCUSSÃO}

A Figura 3 revela detalhes dos tratamentos controle, mineral e organomineral nas doses $40 \%$, $60 \%, 80 \%$ e $100 \%$ do fertilizante mineral. É possível verificar diferenças visuais entre os tratamentos. A produtividade da soja em função da dose e do tipo de fertilizante aplicado é mostrada na Tabela 1 e Figura 4, que revelam uma produtividade da soja com a adubação organomineral de $5,47 \% ; 7,74 \% ; 10,02 \%$ e $17,31 \%$ nas doses de $40 \%, 60 \%, 80 \%$ e $100 \%$, respectivamente, maior do que com a adubação mineral na dose $100 \%$.

Tabela 1 - Produtividade da soja e teor foliar de NPK em função da dose/tipo de fertilizante.

\begin{tabular}{lccccc}
\hline \multicolumn{1}{c}{ Fertilizante } & $\begin{array}{c}\text { Dose } \\
(\mathrm{kg} / \mathrm{ha})\end{array}$ & $\begin{array}{c}\text { Produtividade } \\
(\text { sacas/ha) }\end{array}$ & $\begin{array}{c}\mathrm{N} \\
(\mathrm{g} / \mathrm{kg})\end{array}$ & $\begin{array}{c}\mathrm{P} \\
(\mathrm{g} / \mathrm{kg})\end{array}$ & $\begin{array}{c}\mathrm{K} \\
(\mathrm{g} / \mathrm{kg})\end{array}$ \\
\hline Controle & 0 & $31,9^{(*)}$ & $38,8 \mathrm{~b}$ & $2,3 \mathrm{~b}$ & $18,0 \mathrm{a}$ \\
Mineral (04-20-20) & 400 & $43,9^{(\mathrm{ns})}$ & $38,8 \mathrm{~b}$ & $2,6 \mathrm{ab}$ & $19,0 \mathrm{a}$ \\
Organomineral (03-15-15) & 214 & $46,3^{(\mathrm{n})}$ & $51,3 \mathrm{a}$ & $2,7 \mathrm{ab}$ & $18,8 \mathrm{a}$ \\
Organomineral (03-15-15) & 320 & $47,3^{(\mathrm{ns})}$ & $46,8 \mathrm{ab}$ & $2,5 \mathrm{ab}$ & $16,0 \mathrm{a}$ \\
Organomineral (03-15-15) & 427 & $48,3^{(*)}$ & $48,3 \mathrm{a}$ & $2,8 \mathrm{ab}$ & $17,0 \mathrm{a}$ \\
Organomineral (03-15-15) & 543 & $51,5^{(*)}$ & $51,8 \mathrm{a}$ & $3,1 \mathrm{a}$ & $19,5 \mathrm{a}$ \\
& & $\mathrm{DMS}_{\text {Dunett }}=3,5$ & $\mathrm{DMS}=8,7$ & $\mathrm{DMS}=0,6$ & $\mathrm{DMS}_{\mathrm{K}}=6,5$ \\
& & $\mathrm{CV}=8,3 \%$ & $\mathrm{CV}=10,01 \%$ & $\mathrm{CV}=15,8 \%$ \\
\hline
\end{tabular}

Medias de produtividade seguidas por (*) diferem do tratamento mineral pelo teste de Dunett a $5 \%$ de significância. Médias de teor de NPK seguidas por letras distintas nas colunas diferem entre si pelo teste de Tukey a $5 \%$ de significância. 


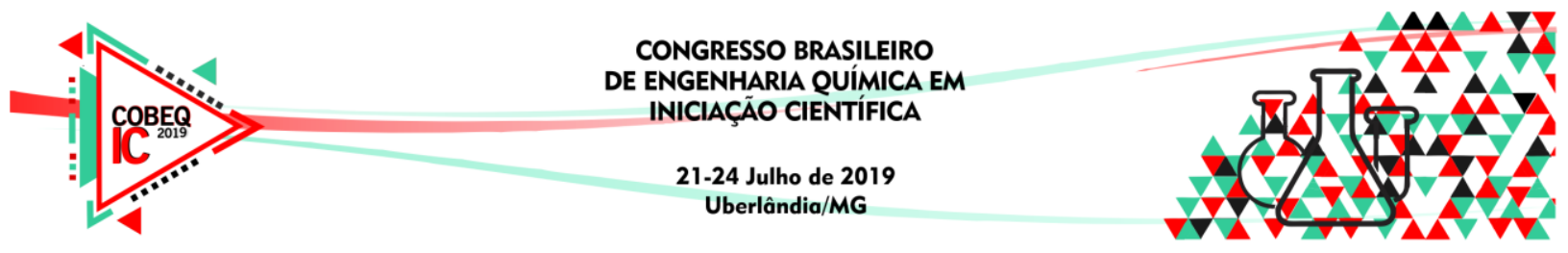

Figura 3 - Foto do local de instalação do experimento. Detalhe dos tratamentos controle, mineral e organomineral nas doses $40 \%, 60 \%, 80 \%$ e $100 \%$ do fertilizante mineral.

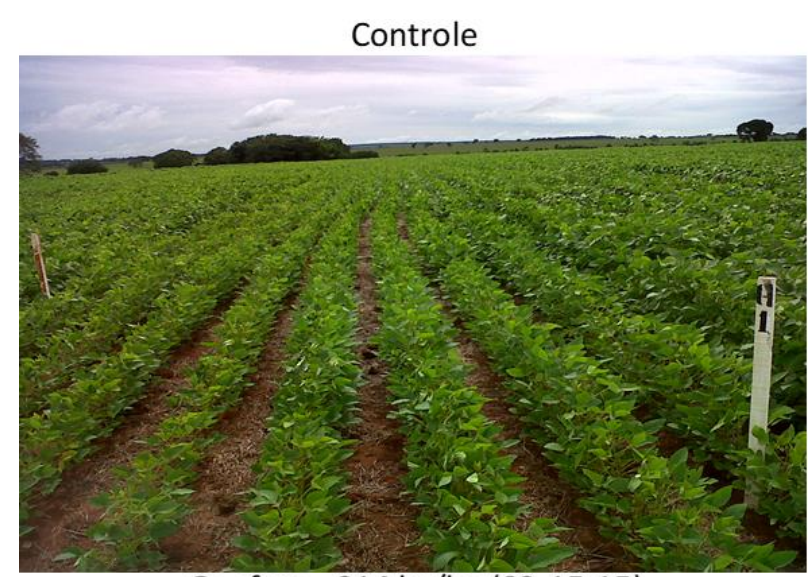

Geofert - $214 \mathrm{~kg} / \mathrm{ha}(03-15-15)$

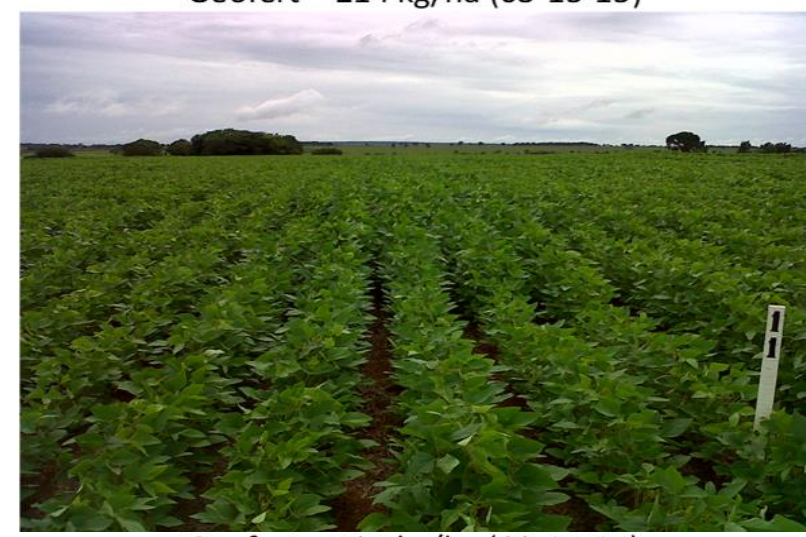

Geofert -427 kg/ha (03-15-15)

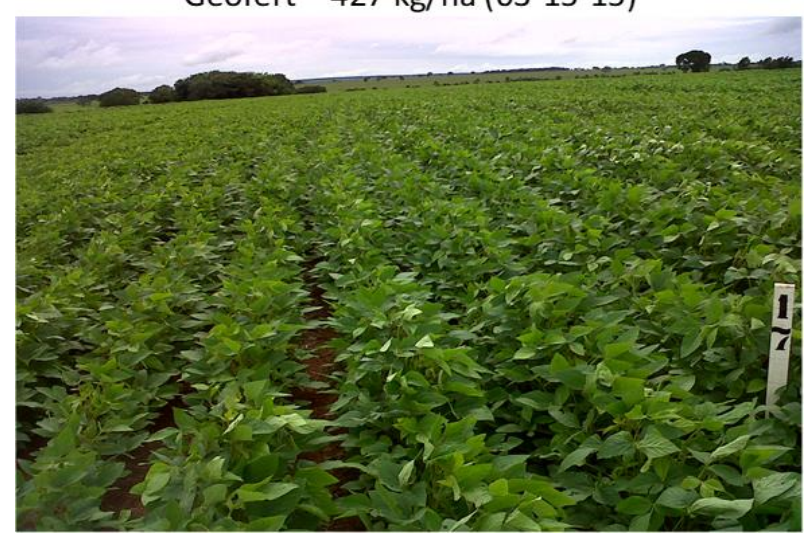

Mineral $-400 \mathrm{~kg} / \mathrm{ha}(4-20-20)$

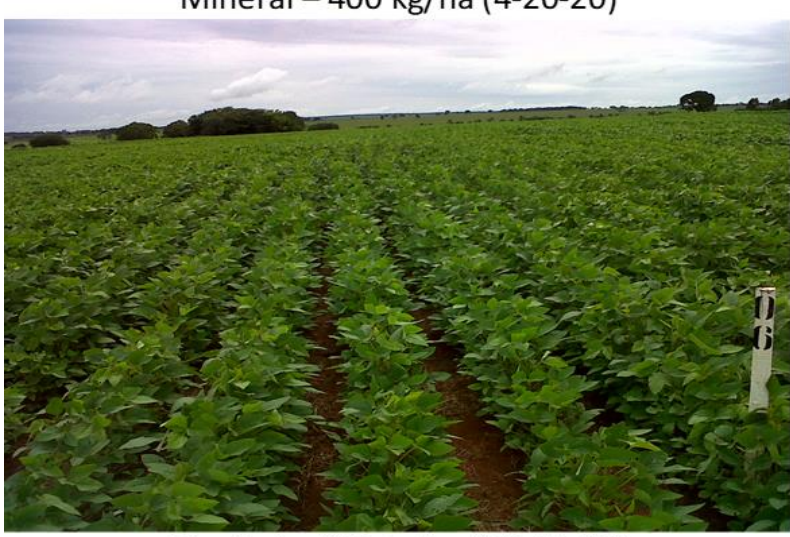

Geofert - 310 kg/ha (03-15-15)

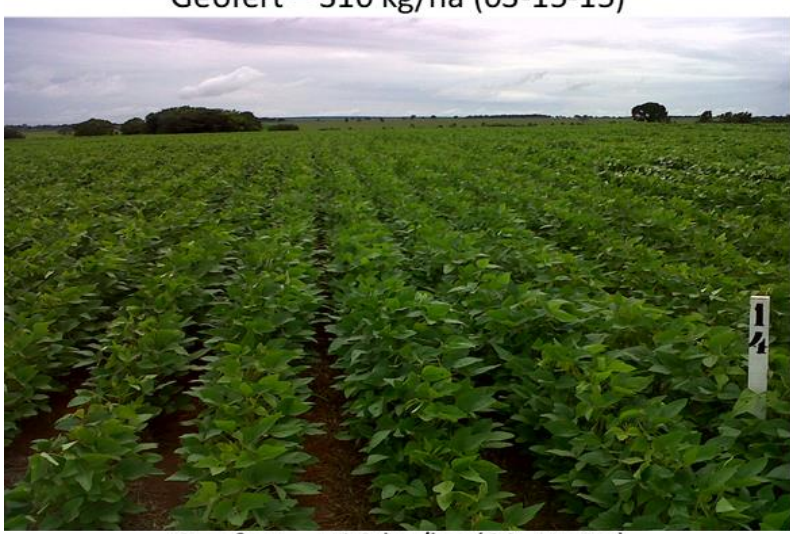

Geofert - 520 kg/ha (03-15-15)

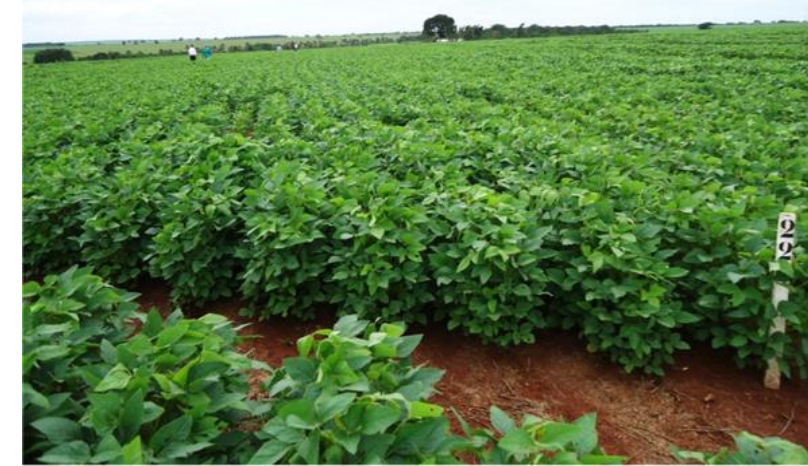

De acordo com a Tabela 1 e a Figura 4 houve diferença estatística em relação à adubação mineral nos tratamentos controle e com organomineral 03-15-15 aplicado nas doses de 427 e $543 \mathrm{~kg} / \mathrm{ha}$, os quais representam 80\% e 100\% de NPK em relação ao tratamento mineral, respectivamente. Além disso, foi verificado incremento na produtividade da soja com a aplicação de doses crescentes do fertilizante organomineral. Já a análise da equação de regressão da produtividade versus dose de organomineral, revelou que uma dosagem $50 \%$ menor do fertilizante organomineral em relação ao fertilizante mineral foi necessária para se 


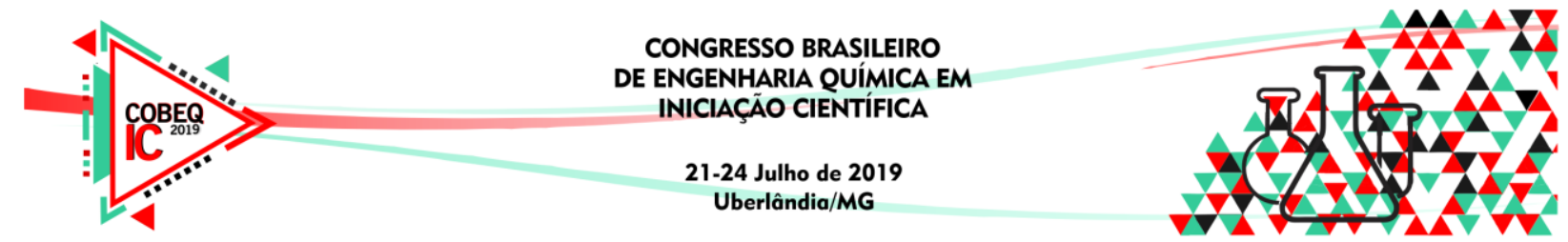

obter a mesma produtividade do fertilizante mineral (Figura 5). Assim, observou-se que com a aplicação de $200 \mathrm{~kg} / \mathrm{ha}$ do organomineral 03-15-15 seria colhido a mesma quantidade de soja obtida com aplicação de $400 \mathrm{~kg} / \mathrm{ha}$ do fertilizante mineral 04-20-20. Com relação os teores de NPK nas folhas foi observado diferenças estatísticas nos teores de N e P em relação aos teores obtidos no tratamento controle e mineral. Para o teor foliar de potássio não houve diferença estatística entre os tratamentos.

Figura 4 - Produtividade da soja em função da dose e do tipo de fertilizante.

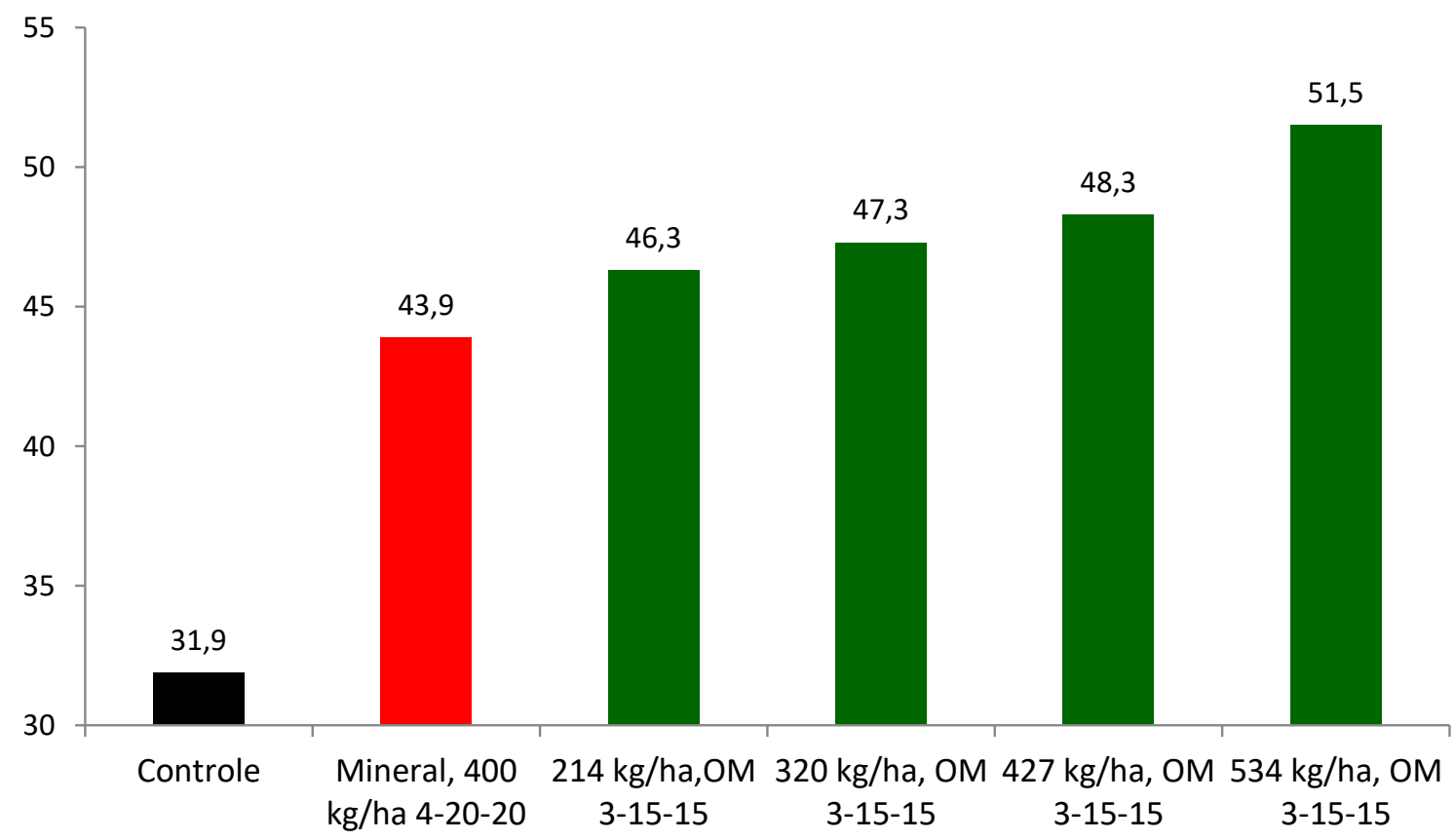

Figura 5 - Análise de regressão da produtividade versus dose de organomineral.

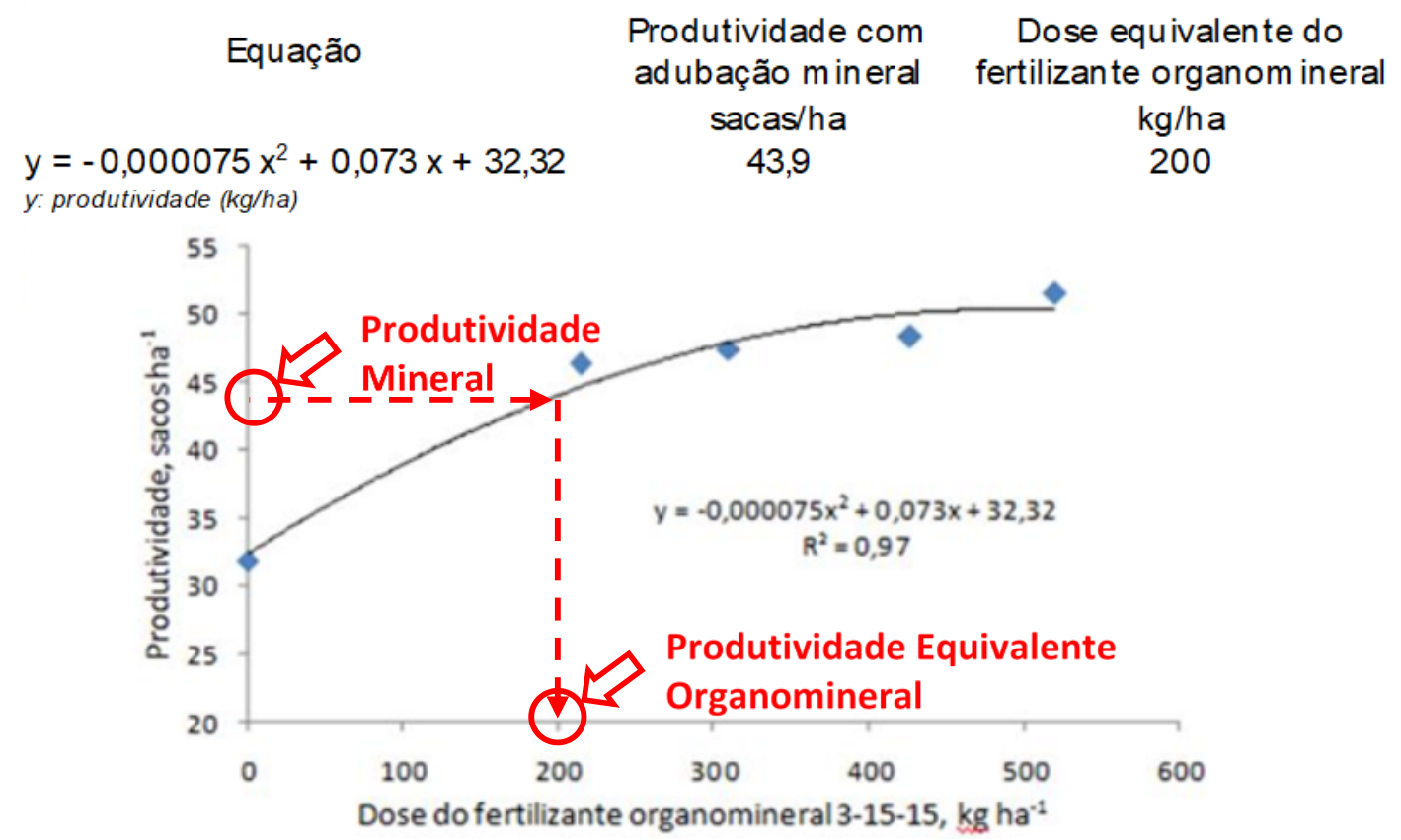




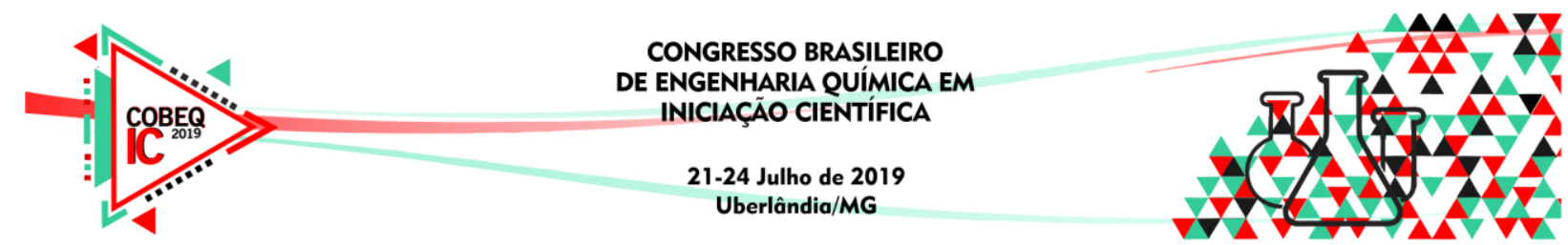

\section{CONCLUSÕES}

A produtividade da soja nos tratamentos que receberam organomineral 03-15-15 foi superior à do tratamento mineral em termos absolutos em todas as doses e, estatisticamente, superior nas doses de 427 e $543 \mathrm{~kg} / \mathrm{ha}$. Existe uma equivalência de produtividade entre 200 $\mathrm{kg} / \mathrm{ha}$ de organomineral 03-15-15 com $400 \mathrm{~kg} / \mathrm{ha}$ de mineral 04-20-20.

\section{AGRADECIMENTOS}

À Finep pelos recursos concedidos, à Geociclo Biotecnologia S/A por fornecer os fertilizantes e a Estância HZ de Tupacigura/MG por ceder a área e os equipamentos para a instalação do experimento.

\section{REFERÊNCIAS}

BHATTACHARYYA R., KUNDU S., PRAKASH V., GRUPTA H.S., Sustainability under combined application of mineral and organic fertilizers in a rainfed soybean wheat system of the Indian Himalayas. European Journal of Agronomy, 28: 33-46, 2008.

FERREIRA D.F., SISVAR: Um programa para análises e ensino de estatística. Revista Symposium, 06: 36-41, 2008.

GHOSH P.K., TRIPATHI, A.K., BANDYOPADHY K.K., MANNA M.C., Assessment of nutrient competition and nutrient requirement in soybean/sorghum intercropping system. European Journal of Agronomy, 31: 43-50, 2009.

SPSS Inc. Released 2008. SPSS Statistics for Windows, Version 17.0. Chicago: SPSS Inc. 